\title{
Balance model for contactless chemo-mechanical polishing of wafers
}

\author{
N.N. Grigoriev, M.Yu. Kravetsky, G.A. Paschenko, S.A. Sypko, A.V. Fomin \\ Institute of Semiconductor Physics, NAS of Ukraine, 45 prospect Nauky, 03028 Kyiv, Ukraine \\ Phone: +380 (44) 265 6189; fax: +380 (44) 265 4110; e-mail: avl@mizar.semicond.kiev.ua
}

\begin{abstract}
We developed a physical model for polishing. It makes it possible to determine physico-chemical processes occurring at contactless chemo-mechanical polishing (CMP) of crystal surfaces. A balance equation for diffusion, convection and chemical flows is used to describe processes that are proceeding in the stationary case. The analytical expressions are obtained that relate polishing rate and surface form for processed material to the physical parameters of the proceeding processes. It was found that macrorelief of the processed surface depends not only on the velocity of polishing plate motion but also on the gap between the processed wafer and polishing plate, as well as active component diffusion in the etching solution. One would expect that, at processing conditions discussed, the surface form is the same for different materials, whatever the active component concentration and chemical reaction constant.

The polishing rate substantially depends on the concentration of the etchant active $\mathrm{co} \mathrm{m}$ ponent, chemical reaction, physical properties of sample material and etching liquid. It is shown that the inverse rate of dissolution is the sum of inverse limiting rates of chemical, diffusion and convection stages of the process. The expressions are obtained that make it possible to optimize technological modes of polishing.
\end{abstract}

Keywords: chemo-mechanical polishing, contactless polishing, polishing plate.

Paper received 25.06.02; accepted for publication 10.12.02.

\section{Introduction}

Formation of flat surfaces for materials used in electronic engineering at final processing stages is usually related to liquid chemical polishing [1]. Either chemo-dynamical or chemo-mechanical versions of this polishing process are applied, depending on the aim to be achieved. Chemo-mechanical polishing (CMP) of flat samples is used when preparing substrates having limiting geometric characteristics. An important feature of the contactless non-abrasion CMP is possibility to form sample surfaces without damaged layer.

For many materials that are used in electronic engineering special etching liquids and polishing modes have been developed. They enable to obtain wafer surfaces having the required geometric parameters. The main of these parameters are as follows: (i) wafer planeness; (ii) «roll-offs» at edges; (iii) degree of parallelism; (iv) material processing tolerance (i.e., a minimal amount of material that has to be removed to obtain surfaces with the required geometric parameters; (v) polishing rate [2]. Due to complexity of physical processes occurring at polish- ing, as well as variety of polishing modifications, only rather few works have been published that dealt with development of physical and analytical models for processes proceeding at polishing of metals semiconductors and insulators [3-7]. At present the CMP technology seems to be an art rather than science, since there exists no unified analytical approach that could enable to find interrelations between physical parameters of the processes involved. Lack of analytical approach to the polishing technology makes the problem of development of new modes and their optimization at variation of technical requirements even more complicated.

The objective of our work is to present a model description of wafer macrorelief formation at contactless CMP and determination of kinetic characteristics of the process involved. This could enable to predict surface form and operation efficiency dependence on technological parameters. Usually during polishing a wafer of processed material executes complex forward and rotary motions relative to the polishing plate. Here we consider a simple case when a plane wafer executes a uniform linear motion. 


\section{N.N. Grigoriev et al.: Balance model for contactless chemo-mechanical polishing of wafers}

\section{Model}

Let us consider a rigid polishing plate as an infinite plane that is moving forward with a constant velocity under surface of a sample of finite length. An etching liquid (in which an active component is dissolved) is on the polishing plate making an even layer. This liquid is brought by the polishing plate under the sample surface. As a result, the sample and polishing plate are separated by a gap that is completely filled with the etchant. The polishing plate material is chemically inert relative to the etchant chosen. Etching of the sample is performed due to interaction between the etchant active component and sample material.

The process of contactless CMP is proceeding in a thin (about several microns) gap between the sample and polishing plate. Liquid motion is forward, and Reynolds numbers are small $(<<1)$. In this case the exact solution for the Navier-Stokes equation for a steady-state laminar flow gives practically linear distribution of stream line velocity over the gap section [8]. The experimental conditions (very small spacing between the wafer and polishing plate) make it possible to present the liquid flow velocity distribution over the gap cross-section in a still simpler form. The etchant flow may be divided into two motionless layers [2], one of which is stuck to the polishing plate and is moving with it, while another layer is stuck to the sample surface. It is supposed that both layers are slipping one relative another at their mutual boundary without friction and do not intermix. Let us denote the etchant layer that is moving with the tool the convection one; and the motionless layer stuck to the sample the diffusion one. The gap width at any point of the sample is retained constant and does not changes during etching. This is an essential feature of polishing process in our experiment.

The etchant active component diffuses from the convection layer along the transverse direction to the diffusion liquid layer. Then the agent diffuses through the motionless layer to the sample surface and enters into chemical reaction with the sample material. This reaction leads to changing the sample surface form.

At the starting moment of etching the spacing between the polishing plate and sample surface is set to be the same along the whole sample length. The solution active component is spent for sample etching. As a result, concentration of this active component is decreasing along the sample. Therefore the initial sample sections are being etched faster, and the spacing between the polished plate and sample surface begins to vary along the sample. Its value at the initial sample sections becomes bigger than that at the sample end where (as it was noted earlier) it remains constant.

The moving layer thickness is assumed to be constant along the whole sample length. So faster etching at the initial sample section leads to increasing the diffusion layer thickness at this sample section. As a result, the supply of the etchant active component to the sample surface is hindered. This fact decreases etching rate for the initial sample section. Therefore, the active component concentration grows at all the gap areas as compared to the earlier moment. Since the gap value at the sample end is fixed, the above fact results in etching rate increase along the sample length. The processes of etching rate decrease for the initial sample sections and its increase along the sample will proceed until its values become the same. Starting from that moment, the polishing process becomes stationary, and a definite sample surface profile is established. In such a steady state any growth of spacing between the polishing plate and profile points (the end point being fixed), say, the initial point, results in decreasing dissolution rate at this place. The rest of profile points are being etched faster, thus restoring the etching rate constancy over the whole sample surface.

Shown in Fig. 1 is the diagram of polishing that is proceeding under stationary conditions. A polishing plate is presented as an infinite plane that is moving with constant linear velocity $U$ under surface of a sample whose length is 1 and width is infinite. The $Y$-axis is oriented at a normal to the polishing plate plane; it lies at the beginning of the sample, along its side face. The $X$-axis lies in the polishing plate plane; it is oriented along the sample length (Fig. 1). The point $x=0$ corresponds to the sample beginning; here it meets the $Y$-axis. At the sample end (at the point $x=l$ ) a gap of constant width $2 \mathrm{~d}$ is kept between the sample and polishing plate. It is presumed that at this point the diffusion layer width is minimal and equal to that of convection layer (in other words, the diffusion layer half-fills the gap). In our model we assume that the side surface at the sample beginning is closed with a membrane that is impervious to the etchant. The membrane base is parallel to the polishing plate plane and is constantly at a distance of $2 d$ from it.

Let concentration of the solution active component at the point of entry into the gap be $c_{0}$. Transport via diffusion occurs along the sample at vertical direction only. It is so intense that the agent concentration in the layer adjacent to the polishing plate is constant at every section $x=x_{s}$; it varies along the horizontal direction only. In the diffusion layer adjacent to the sample the agent concentration decreases linearly, along the vertical di-

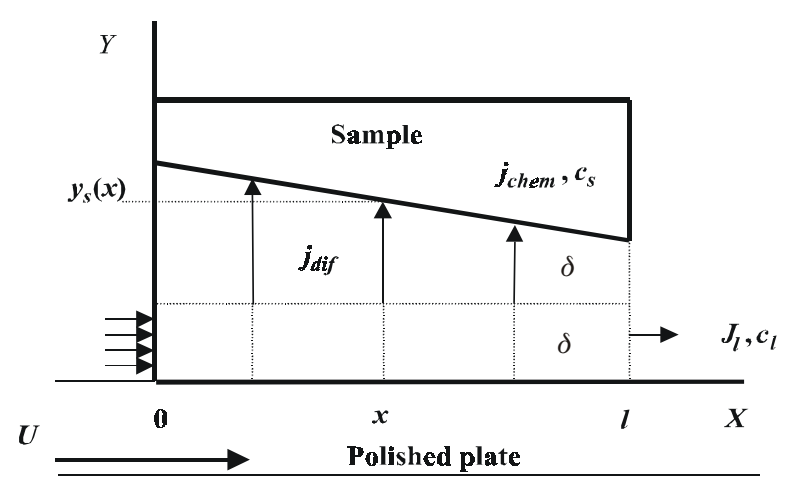

Fig. 1. Model for contactless CMP 
rection, from its maximal value (at the boundary between two layers) down to $c_{s}$ (at the sample surface). And, finally, in our case (stationary etching) the rate of sample dissolution is the same along the whole its length (i.e., the active component concentration at any point of the sample surface is the same: $c_{s}=$ const).

Let the amount of solution active component that is transferred in a unit time across the cross-section be called the flow $j$ of solution active component. Then the flow $j_{0}$ of the agent that enters the gap between the sample and polishing plate is:

$j_{0}=U \delta \cdot c_{0}$.

When moving along the gap, the agent is spent for sample etching, so its concentration is gradually decreased. At the gap outlet it becomes $c_{l}$, and the agent flow going out of the gap is reduced to

$j_{l}=U \delta \cdot c_{l}$.

At every point $x$ at the boundary between the convection and diffusion layers the active component concentration (due to its spending for etching) is $c(x)$. In the layer adjacent to the sample its concentration decreases, along the vertical direction, from the value $c(x)$ (at the boundary between the two layers) to the value $c_{s}$ (at the sample surface). The acting diffusion flow of the agent to the sample surface is:

$j_{D}=D \frac{c(x)-c_{s}}{y_{s}(x)-\delta}$

(here $y_{s}(x)$ is the spacing between the polishing plate and sample surface at the point $x$ ). It is spent for maintenance of chemical reaction (whose reaction constant is $k$ ) between the sample material and etching component. Then the agent flow at a point (the chemical flow) $j_{\text {chem }}$ leading to new surface formation is:

$j_{\text {chem }}=k c_{s}{ }^{n}$.

Generally the exponent $n$ value may be arbitrary, but in our case we shall consider the first-order reaction (i.e., $n=1)$.

The function $y_{1}(x)(3)$ gives the surface profile that is formed due to polishing. Let us determine it from the following considerations.

At the boundary between the convection and diffusion layers the etchant active component passes from the convection layer into the diffusion one, diffuses to the sample and is spent for new surface formation. Under stationary conditions the agent decrease along the $X$-axis is equal to the agent diffusion flow $j$ to the sample surface at the point $x$ :

$U \delta \frac{d c(x)}{d x}=j_{D}=D \frac{c(x)-c_{s}}{y_{s}(x)-\delta}$.

On the other hand,

$$
j_{\text {chem }}=k c_{s}=D \frac{c(x)-c_{s}}{y_{s}(x)-\delta},
$$

so

$y_{s}(x)=\delta+\frac{D\left[c(x)-c_{s}\right]}{k c_{s}}$.

Under stationary conditions

$U \delta \frac{d c(x)}{d x}=j_{\text {chem }}=k c_{s}$.

After integrating this equation, we get the following expression for $c(x)$ :

$c(x)=c_{0}-\frac{k c_{s}}{U \delta} x$.

Thus, according to the model used, the agent concentration linearly decreases along the longitudinal direction. Now let us show that the spacing between the sample surface and boundary between the moving and motionless layers (i.e., $\left.y_{s}(x)\right)$ varies according to just the same law. Indeed, after inserting into expression (8) the $c(x)$ value obtained from (9), we get:

$y_{s}=\delta-\frac{D}{k}-\frac{D c_{0}}{k c_{s}}-\frac{D}{U \delta} x$.

One can eliminate $c_{\mathrm{s}}$ by using the balance equation under stationary conditions and taking proper account of the agent spent for chemical reaction with the sample material. The chemical reaction is proceeding over the whole surface of the sample whose length is $l$, so:

$U \delta \cdot c_{0}=U \delta \cdot c_{l}+k c_{s} \cdot l$,

where $c_{l}$ is the etchant concentration at the gap outlet.

Under stationary conditions the diffusion flow to any surface point is the same and constant:

$j_{D}(x)=D \frac{c(x)-c_{s}}{y_{s}(x)-\delta}=j_{D}(l)=D \frac{c_{l}-c_{s}}{\delta}=k c_{s}$.

From this one gets:

$c_{l}=c_{s}+\frac{k c_{s}}{D} \cdot \delta$

After inserting the above expression for $c_{l}$ into (11), one obtains:

$U \delta \cdot c_{0}=U \delta \cdot\left(c_{s}+\frac{k c_{s}}{D}\right)+k c_{s} \cdot l$

and

$c_{s}=\frac{U \delta \cdot c_{0}}{U \delta+k l+\frac{U \delta^{2} k}{D}}$.

Inserting the obtained expression for $c_{s}(14)$ into (10), we get the equation for profile of the stationary polishing surface: 


\section{N.N. Grigoriev et al.: Balance model for contactless chemo-mechanical polishing of wafers}

$y_{S}(x)=2 \delta+\frac{D}{U \delta} \cdot l-\frac{D}{U \delta} \cdot x=A-b x$,

where

$$
A=2 \delta+\frac{D}{U \delta} l, B=\frac{D}{U \delta} .
$$

Let us determine the rate of sample polishing. Due to chemical interaction between the active component molecules and sample surface, the volume of material that is removed during the time $t$ is such: $W_{m e}=d \cdot l \cdot h$ (here $d$ is the sample width and $h$ is the removed layer thickness). The mass of material that is etched off is $M=\rho_{m e} \cdot W_{m e}=$ $\rho_{m e} d l h$ (here $\rho$ is the sample material density). The reagent mass spent for etching during the same time is $j t d m_{01}$, where $m_{01}$ is mass of the mass of a reagent particle.

Let us define the polishing rate $V_{p o l}$ as thickness of the sample material layer that is removed for the time of polishing $t$; then:

$V_{\text {pol }}=\frac{h}{t}$.

Let one mass unit of the etchant active component is spent for dissolving $\beta$ mass units of the processed material. In this case

$$
\beta j t d \cdot m_{01}=\rho_{m b} d l h .
$$

Then the polishing rate is:

$$
V_{p o l}=\frac{h}{t}=\beta \frac{m_{01}}{\rho_{m b} l} j \text {. }
$$

The expression for the flow $j=j_{\text {chem }}=j_{D}=k \cdot c_{s}$ can be obtained by integrating the equation (8). Then we get the following expression for the diffusion flow at any sample point:

$$
j_{D}=\frac{U \delta}{x} \cdot\left[c_{0}-c(x)\right] .
$$

Under stationary conditions the flow is the same at any point; at the sample end $(x=l)$ it is

$j=j_{D}=\frac{U \delta}{l} \cdot\left(c_{0}-c_{l}\right)$.

On the other hand, $j_{D}=\frac{D}{\delta} \cdot\left(c_{l}-c_{s}\right)$. From this it
llows that $c_{l}=c_{s}+\frac{j_{D} \delta}{D}$.

Let us eliminate $c_{s}$ from this expression using that for the chemical flow: $j=j_{\text {chem }}=j_{D}=k \cdot c_{s}$. Then

$c_{s}=\frac{j}{k}+\frac{j \delta}{D}$.

After inserting this expression into (18), one gets:

$j=\frac{U \delta}{l} \cdot\left(c_{0}-\frac{j}{k}-\frac{j \delta}{D}\right)$.

$S Q O, 5(3), 2002$
Then the agent flow is:

$j=\frac{U \delta}{l} c_{0} /\left(\frac{1}{k}+\frac{\delta}{D}\right)$,

or, for inverse quantity,

$\frac{1}{j}=\frac{1}{c_{0}}\left(\frac{l}{U \delta}+\frac{1}{k}+\frac{\delta}{D}\right)$

Inserting expression (19) for the flow into (17), we finally get for the inverse polishing rate the following expression:

$$
\frac{1}{V_{p o l}}=\frac{\rho}{\beta \cdot c_{0} \cdot m_{01}}\left(\frac{l}{U \delta}+\frac{1}{k}+\frac{\delta}{D}\right) .
$$

\section{Results and discussion}

Our model for etching was developed under the assumption that the active component flows are stationary. No details of diffusion across the convection layer have been taken into account. That is why a term that describes the initial surface region is not represented in expression (15). A more sophisticated consideration of the convection-diffusion processes of surface dissolution will be published elsewhere.

The development of technological conditions required to form surfaces with preset geometric parameters was not the direct objective of this work. It should be noted, however, that (as one can see from expression (15) for the surface profile) it is possible to control departure of sample surface from parallelism by changing diffusion coefficient, velocity of polishing plate motion and gap width (that depends on solution viscosity and velocity of polishing plate motion).

One can see that equation (15) (which describes stationary profile of the sample surface) does not involve those quantities that characterize sample material. The profile of surface obtained does not also depend on the chemical reaction constant and agent concentration; the surface form depends on the parameters $D, U$ and $d$ only.

Thus the obtained sample surface profile (plane) is the same for any material processed if the above polishing technique is applied. (This means the following features: no interaction between agent particles; free access to the reaction surface; free removal of reaction products from the surface; first-order reaction of chemical interaction between agent and sample material.) This fact arises from stability of the polishing process. In our case this stability is achieved by continuous keeping constant width $(2 \delta)$ of the gap between the sample and polishing plate.

Contrary to the above, the polishing rate (20) essentially depends on the properties of both sample material and etching solution. One can see from the form of expression (20) that it is sum of inverse limiting rates of CMP process:

$\frac{1}{V_{p o l}}=\frac{1}{V_{1}}+\frac{1}{V_{2}}+\frac{1}{V_{3}}$, 
where

$$
\begin{aligned}
& \frac{1}{V_{1}}=\frac{\rho l}{U \delta \beta c_{0} m_{01}} ; \\
& \frac{1}{V_{2}}=\frac{\rho}{k \beta c_{0} m_{01}} ; \\
& \frac{1}{V_{3}}=\frac{\rho \delta}{D \beta c_{0} m_{01}} .
\end{aligned}
$$

Evidently the rate of the heterogeneous chemical reaction that determines efficiency of the technique used is limited by the following three factors: (i) etchant transport into the gap between the polishing plate and sample, (ii) diffusion transport of agent molecules to the reaction surface, and (iii) chemical reaction rate. By a limiting rate is meant the rate of such polishing process that is limited by only one of the above factors. To illustrate, if the chemical reaction rate (factor (iii)) is low, then the limiting effect of the factors (i) and (ii) is insufficient and may be neglected. This means that amount of agent that is transported by the polishing plate is much over its consumption due to chemical interaction, and diffusion is proceeding with such high rate that the agent concentration is the same anywhere in the gap and is equal to its initial value. In this case the rate of polishing process depends on the chemical reaction rate only, and this dependence is of the form (24). In two other limiting cases ((i) and (ii)) expressions (22) and (23), respectively, are valid for the process rate.

Let us show that interrelation between the three terms in expression (21) specifies character of the polishing process. We start from the second term that determines the rate of chemical reaction occurring at the sample surface. The constant $a$ of the chemical reaction rate that enters into this term depends, in particular, on the sample surface condition (presence of impurities, dislocations, etc.). As a rule, this condition varies over the sample surface. Therefore, values of the chemical reaction rate are, generally, different at different surface points. So in the case when the second term in expression (21) is comparable to the other two or exceeds them, then it is very probable that different areas of sample surface are dissolved with different rates. This will result in appearance of roughness that makes an undesirable effect. To avoid such an effect, one should apply etching solutions whose constant $k$ is such high that the second term in expression (20) could be neglected.

The first and third terms in expression (20) involve the gap characteristic $\delta$ (the first term involves it in the denominator, while the third one involves it in the numerator). As a result, the function $V_{p o l}(\delta)$ has a peak at a critical value $\delta=\delta_{c r}$. It is easy to understand that if $\delta>\delta_{c r}$, then hollows at the sample surface are dissolving more slowly than projections, i.e., polishing effect would be observed. Contrary to this, at $\delta<\delta_{c r}$ increase of projection height would be observed instead of polishing effect.

\section{Conclusions}

1. We obtained an analytical expression that shows for the sample being polished how its surface form depends on the parameters of polishing process. Stationary surface form depends only on the following physical parameters: coefficient of active component diffusion, velocity of polishing plate motion and gap width. This means that, whatever material nature, one should expect that surfaces of the same form are to be obtained under the above processing conditions.

2. When the velocity of polishing plate motion and gap width are increased, then the slope of the linear area of sample surface goes down, i.e., departure of processed surface from parallelism decreases.

3 . We obtained a theoretical expression for the polishing rate as function of the principal parameters of polishing process. The CMP rate depends on the sample length $l$, type of the chemical reaction used, gap width, properties of both sample material and etching solution, active component concentration. It is shown that, in the case of the first-order chemical reaction, the inverse dissolution rate is equal to the sum of inverse limiting rates of the chemical, diffusion and convection stages of polishing process.

4. The dissolution rate as function of width of the gap between the polishing plate and sample has a peak at $\delta=\delta_{c r}$. At $\delta>\delta_{c r}$ hollows at the sample surface are dissolving more slowly than projections, i.e., polishing effect would be observed. Contrary to this, at $\delta<\delta_{c r}$ increase of projection height rather than polishing effect would be observed.

\section{References}

1. V.G. Levich. Physico-chemical Hydrodynamics, Fizmatgiz, Moscow (1959) (in Russian).

2. C. Rogers, L. Raer, A. Filipossian. Analysis of flow between a wafer and pad during CMP processes. J. Electronic Mater., 27(10), pp. 1082 - 1087 (1998).

3. J. Zabasajia, T. Merchant, Ng Belinda, D. Green. Modeling and characterization of tungsten chemical and mechanical polishing processes. J. Electrochem. Soc., V. 148(2), pp. 673 - 677 (2001).

4. J.M. Steigerwald, S.P. Murarka, R.J. Gutman. Chemical Mechanical Planarization of Microelectronic Materials, Part 1. Wiley and Sons, New York (1997).

5. V.A. Perevozchikov, V.D. Skupov. The Features of Abrasion and Chemical Processing of Semiconductor Surface. Nizhnii Novgorod State Univ. Publ., Nizhnii Novgorod (1992) (in Russian).

6. V.I. Karban', V.V. Rogov, H. Hofman et al. Processing of Semiconductor Materials. Naukova Dumka, Kiev (1982).

7. V.I. Karban', Yu.I. Borzakov. Processing of Single Crystals in Microelectronics. Radio i Svyaz', Moscow (1988).

8. H. Schlichting. Grenzschicht-Theorie. Translation: H. Schlichting. Theory of Boundary Layer. Nauka, Moscow (1974) (in Russian). 